\title{
A NUMERICAL METHOD FOR COMPUTING SINGULAR MINIMIZERS
}

\author{
ZHIPING LI \\ Department of Mathematics, \\ Peking University, \\ Beijing 100871 \\ P.R.CHINA
}

\section{$\S 1$. Introduction}

The main purpose of this paper is to discuss a numerical method, of which a truncation method is a special case, for computing singular minimizers of integrals in the calculus of variations. The idea of trying to find a particular method for detecting singular minimizers is motivated by the so called Lavrentiev phenomenon [1 -5].

Consider the problem of minimizing

$$
I(u)=\int_{0}^{1}\left(u^{3}-x\right)^{2}\left(u^{\prime}\right)^{6} d x
$$

in the set of admissible functions

$$
\mathbf{A}=\left\{u \in W^{1,1}(0,1): u(0)=0, u(1)=1\right\}
$$

It is easy to see that the unique minimizer of $I$ in $\mathbf{A}$ is $\hat{u}=x^{1 / 3}$ and that $I(\hat{u})=0$. It was shown by Manià $[2]$ that the Lavrentiev phenomenon occurs in the problem, i.e.

$$
\inf _{u \in \mathbf{A} \cap W^{1, \infty}(0,1)} I(u)>\inf _{u \in \mathbf{A}}=I(\hat{u})=0
$$

Furthermore, Ball \& Mizel [3] showed that if $p \geq 3 / 2$

$$
\lim _{i \rightarrow \infty} I\left(u_{i}\right)=\infty
$$

Typeset by $\mathcal{A} \mathcal{M} \mathcal{S}-\mathrm{T}_{\mathrm{E}} \mathrm{X}$ 
for any sequence of functions $u_{i} \in \mathbf{A} \cap W^{1, p}(0,1)$ converging almost everywhere to $\hat{u}$. It is easily seen from (1.3) and (1.4) that any numerical method based on a sufficiently accurate computation of $I\left(u_{i}\right)$ for Lipschitz functions $u_{i}$ will fail both to locate $\hat{u}$ and to produce the correct minimum value of $I$ in $\mathbf{A}$.

The existing numerical methods, which can avoid Lavrentiev phenomenon and detect singular minimizers, can be found in $[4,6]$. To apply truncation methods to compute singular minimizers was suggested by J.M.Ball.

In this paper, a numerical method with a more general form, which includes truncation methods as special cases, is described (§3). As a theoretical base of the method, some lower semicontinuity theorems [7] are given in $\S 2$. Approximation properties and convergence theorems of the method are established in $\S 3$. In $\S 4$, I describe 2 truncation methods as examples of the method given in $\S 3$. In $\S 5$, I show the results of a numerical example.

\section{$\S 2$. Lower semicontinuity theorems}

Let $\Omega \subset R^{n}$ be bounded and open.

Definition 2.1. A function $f: \Omega \times R^{m} \times R^{k} \rightarrow R$ is a Carathéodory function if

(1) $f(\cdot, u, P)$ is measurable for every $u \in R^{m}, P \in R^{k}$,

(2) $f(x, \cdot, \cdot)$ is continuous for almost every $x \in \Omega$.

Throughout the rest of this paper $\rightarrow$ denotes the weak convergence of sequences. The following theorems are special cases, where $u$ and $P$ are related by $P=D u$, of a general lower semicontinuity theorem given by Li $[7]$.

Theorem 2.1. Let $f: \Omega \times R^{m} \times R^{m \times n} \rightarrow R$ satisfy

( i ) $f(x, u, P)$ is a Carathéodory function;

( ii) $f(x, u, P) \geq a(x), a(x) \in L^{1}(\Omega)$;

(iii) $f(x, u, \cdot)$ is convex.

Let $f_{M}: \Omega \times R^{m} \times R^{m \times n} \rightarrow R$ satisfy

(a) $f_{M}(x, u, P)$ are Carathéodory functions;

(b) $f_{M}(x, u, P) \geq a(x), \quad a(x) \in L^{1}(\Omega)$;

(c) There exists a sequence of compact subsets $\Omega_{l}$ in $\Omega$ such that

$$
\lim _{l \rightarrow \infty} \operatorname{meas}\left(\Omega \backslash \Omega_{l}\right)=0
$$

and

$$
f_{M} \longrightarrow f, \quad \text { uniformly on } \Omega_{l} \times G \text {, }
$$


for each $l$ and any compact set $G$ in $R^{m} \times R^{m \times n}$.

Let $\left\{u_{M}\right\}, u \in W^{1, p}\left(\Omega ; R^{m}\right), 1 \leq p \leq \infty$ be such that

$$
u_{M} \rightarrow u, \text { in } W^{1, p}\left(\Omega ; R^{m}\right)
$$

Then

$$
I(u) \leq \underline{\lim }_{M \rightarrow \infty} I_{M}\left(u_{M}\right)
$$

where

$$
\begin{aligned}
I(u) & =\int_{\Omega} f(x, u, D u) d x, \\
I_{M}(v) & =\int_{\Omega} f_{M}(x, v, D v) d x .
\end{aligned}
$$

Theorem 2.2. Let $f$ satisfy (i) - (iii) in theorem 2.1. Let $\left\{f_{M}\right\}$ satisfy (a), (b) in theorem 2.1 and

$\left(c^{\prime}\right)$ There exists a sequence of compact subsets $\Omega_{l}$ in $\Omega$ such that

$$
\lim _{l \rightarrow \infty} \operatorname{meas}\left(\Omega \backslash \Omega_{l}\right)=0
$$

and

$\int_{\Omega_{l} \backslash E(v, K)}\left|f_{M}(x, v, D v)-f(x, v, D v)\right| d x \longrightarrow 0, \quad$ uniformly in $W^{1, p}\left(\Omega ; R^{m}\right)$,

for each $l$ and any fixed $K>0$, where $E(v, K)=\{x \in \Omega:|v|>$ $K$, or $|D v|>K\}$.

Let $\left\{u_{M}\right\}, u \in W^{1, p}\left(\Omega ; R^{m}\right), 1 \leq p \leq \infty$, satisfy

$$
u_{M} \rightarrow u, \text { in } W^{1, p}\left(\Omega ; R^{m}\right) .
$$

Then

$$
I(u) \leq \underline{\lim }_{M \rightarrow \infty} I_{M}\left(u_{M}\right)
$$




\section{$\S 3$. The method and its properties}

Assume for simplicity that $\Omega \subset R^{n}$ is a polyhedron and $\partial \Omega_{0} \subset \partial \Omega$, where $\partial \Omega$ is the boundary of $\Omega$, consists of faces of the polyhedron. Let $T_{h}$ be regular triangulations of $\Omega$ with $h$ being the mesh size [8]. Let

$$
\begin{aligned}
A\left(u_{0} ; \partial \Omega_{0}\right) & =\left\{u \in W^{1, p}\left(\Omega ; R^{m}\right): u=u_{0}, \text { on } \partial \Omega_{0}\right\} ; \\
A_{h} & =\left\{u \in C(\bar{\Omega}): u \text { is a polynomial of degree } \mathrm{k} \text { on } K, \forall K \in T_{h}\right\} ; \\
A_{h}\left(u_{0 h} ; \partial \Omega_{0}\right) & =\left\{u \in A_{h}: u=u_{0 h} \text { on } \partial \Omega_{0}\right\},
\end{aligned}
$$

where $u_{0 h} \in A_{h}$ satisfy

$$
u_{0 h} \longrightarrow u_{0}, \text { in } W^{1, p}\left(\Omega ; R^{m}\right)
$$

Our method for computing the minimizers of $I(u)=\int_{\Omega} f(x, u, D u) d x$ in $A\left(u_{0} ; \partial \Omega_{0}\right)$ is to solve the finite problem of minimizing $I_{M}\left(u_{h}\right)=\int_{\Omega} f_{M}(x$, $\left.u_{h}, D u_{h}\right) d x$ in $A_{h}\left(u_{0 h} ; \partial \Omega_{0}\right)$ for properly chosen $f_{M}$ and $h$.

Lemma 3.1. Let $f: \Omega \times R^{m} \times R^{m \times n} \rightarrow R$ satisfy

( i ) $f(x, u, P)$ is a Carathéodory function;

( ii) $f(x, u, P) \geq a(x), a(x) \in L^{1}(\Omega)$;

Let $f_{M}: \Omega \times R^{m} \times R^{m \times n} \rightarrow R$ satisfy

(a) $f_{M}(x, u, P)$ are Carathéodory functions;

(b) $a(x) \leq f_{M}(x, u, P) \leq \min \left\{f(x, u, P), b_{M}(x)+a_{M}(x)\left(|u|^{p}+|P|^{p}\right)\right\}$, where $a(x), b_{M}(x) \in L^{1}(\Omega), a_{M}(x) \in L^{\infty}(\Omega)$ and $1 \leq p \leq \infty$;

$\left(c^{\prime \prime}\right)$ For any $v \in W^{1, p}\left(\Omega ; R^{m}\right)$

$$
f_{M}(x, v, D v) \longrightarrow f(x, v, D v), \text { in measure }
$$

i.e. for any $\epsilon>0$

meas $\left\{x \in \Omega:\left|f_{M}(x, v, D v)-f(x, v, D v)\right| \geq \epsilon\right\} \rightarrow 0$, as $M \rightarrow \infty$.

Let $u \in A\left(u_{0} ; \partial \Omega_{0}\right)$ be such that $f(x, u(x), D u(x)) \in L^{1}(\Omega)$. Let $u_{h} \in$ $A_{h}\left(u_{0 h} ; \partial \Omega_{0}\right)$

be such that

$$
u_{h} \longrightarrow u, \quad \text { in } W^{1, p}\left(\Omega ; R^{m}\right) .
$$

Then, for any $\epsilon>0$, there exist $M(\epsilon)>0$ and $h(\epsilon, M)>0$ such that

$$
\left|I_{M}\left(u_{h}\right)-I(u)\right|<\epsilon, \text { for } M>M(\epsilon) \text { and } 0<h<h(\epsilon, M) .
$$


Proof.

$$
\begin{aligned}
& I_{M}\left(u_{h}\right)-I(u) \\
= & \int_{\Omega}\left(f_{M}\left(x, u_{h}, D u_{h}\right)-f_{M}(x, u, D u)\right) d x \\
& +\int_{\Omega}\left(f_{M}(x, u, D u)-f(x, u, D u)\right) d x \\
= & I_{1}+I_{2}
\end{aligned}
$$

It follows from $f(x, u(x), D u(x)) \in L^{1}(\Omega)$ and $a(x) \in L^{1}(\Omega)$ that for any $\epsilon>0$ there exists $\delta(\epsilon)>0$ such that

$$
\int_{\Omega^{\prime}}|f(x, u(x), D u(x))| d x<\epsilon
$$

and

$$
\int_{\Omega^{\prime}}|a(x)| d x<\epsilon,
$$

for any $\Omega^{\prime} \subset \Omega$ with meas $\left(\Omega^{\prime}\right)<\delta(\epsilon)$.

On the other hand, let

$$
\begin{aligned}
& \Omega_{M}(\epsilon)= \\
& \left\{x \in \Omega:\left|f_{M}(x, u(x), D u(x))-f(x, u(x), D u(x))\right|>\epsilon /(8 \operatorname{meas}(\Omega))\right\} .
\end{aligned}
$$

Then, by $\left(c^{\prime \prime}\right)$, for any $\epsilon>0$ and $\delta>0$ there exists $M(\epsilon, \delta)>1$ such that

$$
\operatorname{meas}\left(\Omega_{M}(\epsilon)\right)<\delta, \quad \forall M \geq M(\epsilon, \delta) .
$$

Now, by taking $M(\epsilon)=M(\epsilon / 8, \delta(\epsilon / 8))$ and by using the fact (see (b)) that

$$
\left|f_{M}(x, u(x), D u(x))\right| \leq|a(x)|+|f(x, u(x), D u(x))|,
$$

we have

$$
\begin{aligned}
\left|I_{2}\right| \leq & \int_{\Omega_{M}(\epsilon)}(|a(x)|+2|f(x, u(x), D u(x))|) d x \\
& +\int_{\Omega \backslash \Omega_{M}(\epsilon)}\left|f_{M}(x, u, D u)-f(x, u, D u)\right| d x \\
\leq & 4 \cdot \epsilon / 8=\epsilon / 2, \quad \forall M \geq M(\epsilon) .
\end{aligned}
$$

We claim that for any $\epsilon>0$ and $M>0$ there exists $h(\epsilon, M)>0$ such that

$$
\left|I_{1}\right|<\epsilon / 2, \quad \forall 0<h \leq h(\epsilon, M) .
$$


Suppose otherwise. Then, there would be $\epsilon_{0}>0, M_{0}>0$ and a decreasing sequence $\left\{h_{j}\right\}$ with $\lim _{j \rightarrow \infty} h_{j}=0$ such that

$$
\left|\int_{\Omega}\left(f_{M_{0}}\left(x, u_{h_{j}}, D u_{h_{j}}\right)-f_{M_{0}}(x, u, D u)\right) d x\right| \geq \epsilon_{0} / 2, \quad \forall j .
$$

By (3.2), we may assume

$$
\begin{array}{r}
u_{h_{j}} \longrightarrow u, \text { almost everywhere in } \Omega, \\
D u_{h_{j}} \longrightarrow D u, \text { almost everywhere in } \Omega .
\end{array}
$$

Thus by (a)

$$
f_{M_{0}}\left(x, u_{h_{j}}, D u_{h_{j}}\right) \rightarrow f_{M_{0}}(x, u, D u), \text { almost everywhere in } \Omega \text {. }
$$

On the other hand, by (b)

$$
\begin{aligned}
& \left|f_{M_{0}}\left(x, u_{h_{j}}, D u_{h_{j}}\right)-f_{M_{0}}(x, u, D u)\right| \\
\leq & b_{M_{0}}(x)+a_{M_{0}}(x)\left(\left|u_{h_{j}}\right|^{p}+|u|^{p}+\left|D u_{h_{j}}\right|^{p}+|D u|^{p}\right) .
\end{aligned}
$$

By (3.2), the right hand side of (3.7) is uniformly integral continuous. Hence by (3.6) and (3.7),

$$
\left|I_{1}\right| \longrightarrow 0, \text { as } j \rightarrow \infty
$$

This is a contradiction.

(3.3) now follows from (3.4) and (3.5).

As a direct corollary of lemma 3.1, we have

Theorem 3.1. Let $f, f_{M}$ satisfy the hypotheses in lemma 3.1. Then, for any $\epsilon>0$, there exist $M(\epsilon)>0$ and $h(\epsilon, M)>0$ such that

$$
\begin{aligned}
& \inf _{u_{h} \in A_{h}\left(u_{0 h} ; \partial \Omega_{0}\right)} I_{M}\left(u_{h}\right)< \\
& \quad \inf _{u \in A\left(u_{0} ; \partial \Omega_{0}\right)} I(u)+\epsilon, \\
& \quad \text { for } M \geq M(\epsilon), 0<h \leq h(\epsilon, M) .
\end{aligned}
$$

Now, we can prove the following convergence theorem for the method.

Theorem 3.2. Let $1<p<\infty$. Let $f: \Omega \times R^{m} \times R^{m \times n} \rightarrow R$ satisfy

( i ) $f(x, u, P)$ is a Carathéodory function;

( ii) $f(x, u, P) \geq a(x), a(x) \in L^{1}(\Omega)$;

(iii) $f(x, u, \cdot)$ is convex. 
Let $f_{M}: \Omega \times R^{m} \times R^{m \times n} \rightarrow R$ satisfy

(a) $f_{M}(x, u, P)$ are Carathéodory functions;

(b) $a(x) \leq f_{M}(x, u, P) \leq \min \left\{f(x, u, P), b_{M}(x)+a_{M}(x)\left(|u|^{p}+|P|^{p}\right)\right\}$, where $a(x), b_{M}(x) \in L^{1}(\Omega), a_{M}(x) \in L^{\infty}(\Omega)$;

$\left(c^{\prime}\right)$ There exists a sequence of compact subsets $\Omega_{l}$ in $\Omega$ such that

$$
\lim _{l \rightarrow \infty} \text { meas }\left(\Omega \backslash \Omega_{l}\right)=0
$$

and

$$
\begin{aligned}
& \int_{\Omega_{l} \backslash E(v, K)}\left|f_{M}(x, v, D v)-f(x, v, D v)\right| d x \longrightarrow 0, \\
& \text { uniformly in } W^{1, p}\left(\Omega ; R^{m}\right)
\end{aligned}
$$

for each $l$ and any fixed $K>0$, where $E(v, K)=\{x \in \Omega:|v|>$ $K$, or $|D v|>K\}$.

Let $\left\{\epsilon_{j}\right\}$ be a decreasing sequence satisfying $\lim _{j \rightarrow \infty} \epsilon_{j}=0$. Let $M_{j}=M\left(\epsilon_{j}\right)$ and $h_{j}=h\left(\epsilon_{j}, M_{j}\right)$, where $M\left(\epsilon_{j}\right)$ and $h\left(\epsilon_{j}, M_{j}\right)$ are valued by theorem 3.1. Let $u_{h_{j}} \in A_{h_{j}}\left(u_{0 h_{j}} ; \partial \Omega_{0}\right)$ be such that

$$
I_{j}\left(u_{j}\right)=\int_{\Omega} f_{M_{j}}\left(x, u_{j}, D u_{j}\right) d x<\inf _{v \in A_{h_{j}}\left(u_{0 h_{j}} ; \partial \Omega_{0}\right)} I_{j}(v)+\epsilon_{j}
$$

and

$$
u_{0 h_{j}} \longrightarrow u_{0}, \quad \text { in } W^{1, p}\left(\Omega ; R^{m}\right) .
$$

Assume $\left\{u_{j}\right\}$ are uniformly bounded in $W^{1, p}\left(\Omega ; R^{m}\right)$, i.e. there is a constant $C>0$ such that

$$
\left\|u_{j}\right\|_{1, p} \leq C, \quad \forall j
$$

Then, there exists a function $u \in A\left(u_{0} ; \partial \Omega_{0}\right)$ and a subsequence of $\left\{u_{j}\right\}$, again denoted $\left\{u_{j}\right\}$, such that

$$
u_{j} \rightarrow u, \quad \text { in } W^{1, p}\left(\Omega ; R^{m}\right),
$$

and

$$
I(u)=\inf _{v \in A\left(u_{0} ; \partial \Omega_{0}\right)} I(v)=\underline{\lim }_{j \rightarrow \infty} I_{j}\left(u_{j}\right) .
$$

Proof. It is a classical result that (3.11) holds for a function $u \in W^{1, p}(\Omega$; $\left.R^{m}\right)$. From (3.9), it follows that $u=u_{0}$ on $\partial \Omega_{0}$, i.e. $u \in A\left(u_{0} ; \partial \Omega_{0}\right)$. 
By theorem 2.2, we have

$$
I(u) \leq \underline{\lim }_{j \rightarrow \infty} I_{j}\left(u_{j}\right) .
$$

It follows from $\left(c^{\prime}\right)$ that $f_{M}$ also satisfy $\left(c^{\prime \prime}\right)$ in lemma 3.1. Hence by theorem 3.1, we have

$$
\underline{\lim }_{j \rightarrow \infty} I_{j}\left(u_{j}\right) \leq \inf _{v \in A\left(u_{0} ; \partial \Omega_{0}\right)} I(v) .
$$

Combining (3.13) and (3.14), we have (3.12).

Remark. If $\left(c^{\prime}\right)$ is replaced by (c) in theorem 3.2, the conclusion of the theorem still holds.

\section{$\S 4$. Truncation methods}

To apply the results in $\S 2$ and $\S 3$, we need to find an appropriate sequence of functions $\left\{f_{M}\right\}$. We may use the truncation method to construct such sequences.

Let $1<p<\infty$ and define

$$
\bar{f}_{M}(x, u, P)= \begin{cases}f(x, u, P), & \text { if }|u| \leq M \text { and }|P| \leq M \\ f\left(x, u_{M}, P_{M}\right)+ & \alpha_{M}(x)\left(\chi_{M}^{p}(|u|)+\chi_{M}^{p}(|P|)\right), \text { otherwise }\end{cases}
$$

where

$$
\begin{gathered}
u_{M}=\left\{\begin{array}{lc}
u, & \text { if }|u| \leq M ; \\
\frac{M}{|u|} u, & \text { if }|u|>M,
\end{array}\right. \\
P_{M}=\left\{\begin{array}{lc}
P, & \text { if }|P| \leq M ; \\
\frac{M}{|P|} P, & \text { if }|P|>M,
\end{array}\right. \\
\chi_{M}^{p}(t)=\left\{\begin{array}{lr}
0, & \text { if } t \leq 0 ; \\
t^{p}-M^{p}, & \text { if } t>0,
\end{array}\right.
\end{gathered}
$$

and

$$
\alpha_{M} \in L^{\infty}, \quad \alpha_{M}(x) \geq c>0, \forall x \in \Omega
$$

Define

$$
f_{M}^{1}(x, u, P)=\min \left\{f(x, u, P), \bar{f}_{M}(x, u, P)\right\} .
$$

Let $\left\{\bar{\alpha}_{M}\right\}$ be an increasing sequence satisfying

$$
\bar{\alpha}_{1} \geq c>0, \lim _{M \rightarrow \infty} \bar{\alpha}_{M}=\infty
$$

Define

$$
f_{M}^{2}(x, u, P)=\min \left\{f(x, u, P), \bar{\alpha}_{M}\left(1+|P|^{p}\right)\right\}
$$


Lemma 4.1. Let $f: \Omega \times R^{m} \times R^{m \times n} \rightarrow R$ satisfy

( i ) $f(x, u, P)$ is a Carathéodory function;

( ii) $f(x, u, P) \geq a(x), a(x) \in L^{1}(\Omega)$;

(iii) $f(x, u, \cdot)$ is convex.

(iv) Let $g_{K}(x)=\sup _{|u| \leq K,|P| \leq K}|f(x, u, P)|$. Then $g_{K}(\cdot) \in L^{1}(\Omega)$.

Then, $f_{M}^{i}, i=1,2$, defined by (4.2) and (4.4) respectively, satisfy

(a) $f_{M}^{i}(x, u, P)$ are Carathéodory functions;

(b) $\bar{a}(x) \leq f_{M}^{i}(x, u, P) \leq \min \left\{f(x, u, P), b_{M}(x)+a_{M}(x)\left(|u|^{p}+|P|^{p}\right)\right\}$, where $\bar{a}(x), b_{M}(x) \in L^{1}(\Omega), a_{M}(x) \in L^{\infty}(\Omega), a_{M}(x) \geq c>0$, a.e. in $\Omega$.

and furthermore $f_{M}^{1}$ satisfy

(c) There exists a sequence of compact sets $\Omega_{l}$ in $\Omega$ such that

$$
\lim _{l \rightarrow \infty} \text { meas }\left(\Omega \backslash \Omega_{l}\right)=0
$$

and

$$
f_{M}^{1} \longrightarrow f, \quad \text { uniformly on } \Omega_{l} \times G
$$

for each $l$ and any compact set $G \in R^{m} \times R^{m \times n}$;

$f_{M}^{2}$ satisfy

$\left(c^{\prime}\right)$ There exists a sequence of compact sets $\Omega_{l}$ in $\Omega$ such that

$$
\lim _{l \rightarrow \infty} \text { meas }\left(\Omega \backslash \Omega_{l}\right)=0
$$

and

$\int_{\Omega_{l} \backslash E(v, K)}\left|f_{M}^{2}(x, v, D v)-f(x, v, D v)\right| d x \longrightarrow 0$

uniformly in $W^{1, p}\left(\Omega ; R^{m}\right)$,

for each $l$ and any fixed $K>0$ where $E(v, K)=\{x \in \Omega:|v|>$ $K$, or $|D v|>K\}$.

Proof. (a) is obvious.

For $i=1$, take $\bar{a}(x)=a(x), b_{M}(x)=g_{M}(x)$ (see (iv)) and $a_{M}(x)=$ $\alpha_{M}(x)$. For $i=2$, take $\bar{a}(x)=\min \left\{a(x), \alpha_{1}\right\}$, and $a_{M}(x)=b_{M}(x)=\bar{\alpha}_{M}$. Then, it is easy to check that (b) holds.

It is obvious that (c) is satisfied by $f_{M}^{1}$, since $f_{M}(x, u, P)=f(x, u, P)$ for all $x \in \Omega,|u| \leq M$ and $|P| \leq M$.

Now we show that $\left(c^{\prime}\right)$ is satisfied by $f_{M}^{2}$. 
For any $\epsilon>0$ and $K>1$, it follows from (iv) that there exist $C(K)>0$ and $\delta(\epsilon, K)>0$ such that

$$
\int_{\Omega} g_{K}(x) \leq C(K)
$$

and

$$
\int_{\Omega^{\prime}} g_{K}(x) \leq \epsilon, \forall \Omega^{\prime} \subset \Omega \text { with meas }\left(\Omega^{\prime}\right)<\delta(\epsilon, K),
$$

where $g_{K}$ is defined as in (iv).

For any $\delta>0$ and $K>1$, it follows from (4.5) that there exists $A(K, \delta)>$ 1 such that

$$
\text { meas }(G(K, A))<\delta, \quad \forall A \geq A(K, \delta),
$$

where $G(K, A)=\left\{x \in \Omega: g_{K}(x)>A\right\}$.

By (4.3), for any $A>0$ there exists $M(A)>1$ such that

$$
\bar{\alpha}_{M} \geq A, \quad \forall M \geq M(A) .
$$

By (4.4) and (4.8),

$$
\begin{aligned}
f_{M}^{2}(x, v(x), D v(x))= & f(x, v(x), D v(x)), \\
& \forall x \in \Omega \backslash G(K, A), \text { and } \forall M \geq M(A) .
\end{aligned}
$$

Thus, by taking $\delta=\delta(\epsilon, K), A=A(\epsilon, K)=A(K, \delta(\epsilon, K))$, and $M(\epsilon, K)=$ $M(A(\epsilon, K))$ and by (4.4), (4.6), (4.7) and (4.9), we have

$$
\begin{aligned}
& \int_{\Omega \backslash E(v, K)}\left|f_{M}^{2}(x, v, D v)-f(x, v, D v)\right| d x \\
= & \int_{(\Omega \backslash E(v, K)) \cap G(K, A)}\left|f_{M}^{2}(x, v, D v)-f(x, v, D v)\right| d x \\
\leq & 2 \int_{(\Omega \backslash E(v, K)) \cap G(K, A)}|f(x, v, D v)| d x \\
\leq & 2 \int_{G(K, A)} g_{K}(x) d x \\
< & 2 \epsilon, \quad \forall M \geq M(\epsilon, K) \text { and } \quad v \in W^{1, p}\left(\Omega ; R^{m}\right) .
\end{aligned}
$$

(4.10) implies that $f_{M}^{2}$ satisfy $\left(c^{\prime}\right)$.

By lemma 4.1, we know that all the results in $\S 2$ and $\S 3$ remain valid if $f_{M}$ is substituted by $f_{M}^{1}$ defined by (4.2) or $f_{M}^{2}$ defined by (4.4). 
Let $\Omega, \partial \Omega_{0}$ and $T_{h}$ be the same as in $\S 3$. Let

$$
A=\left\{u \in W^{1,1}\left(\Omega ; R^{m}\right): u=0, \quad \text { on } \partial \Omega_{0}\right\},
$$

$A_{h}=\left\{u \in C(\bar{\Omega}): u\right.$ is a polynomial of degree $\leq k$ on each element in $T_{h}$, $u=0$ on $\left.\partial \Omega_{0}\right\}$

Suppose $\hat{u} \in W^{1, p}\left(\Omega ; R^{m}\right), p>1$, is a minimizer of

$$
I(u)=\int_{\Omega} f(x, u, D u) d x
$$

in $A$. Let

$$
\begin{aligned}
L & =1+|\hat{u}|_{1, p}^{p}, \\
A_{h}(L) & =\left\{u \in A_{h}: \int_{\Omega}|D u|^{p} d x \leq L\right\} .
\end{aligned}
$$

Theorem 4.1. Let $f: \Omega \times R^{m} \times R^{m \times n} \rightarrow R$ satisfy

( i ) $f(x, u, P)$ is a Carathéodory function;

(ii) $f(x, u, P) \geq a(x), a(x) \in L^{1}(\Omega)$;

(iii) $f(x, u, \cdot)$ is convex.

(iv) Let $g_{K}(x)=\sup _{|u| \leq K,|P| \leq K}|f(x, u, P)|$. Then $g_{K}(\cdot) \in L^{1}(\Omega)$.

Let $f_{M}^{i}, i=1,2$, be defined by (4.2) and (4.4) respectively. Let $\left\{\epsilon_{j}\right\}$ be a decreasing sequence with $\lim _{j \rightarrow \infty} \epsilon_{j}=0$. We have

(1) There exist a nonincreasing function $M(\epsilon)>0$ and a function $h(\epsilon, M)$ with $h(\cdot, M)$ nondecreasing and $h(\epsilon, \cdot)$ nonincreasing such that

$$
\inf _{v \in A_{h}(L)} I_{M}^{i}(v)<I(\hat{u})+\epsilon_{j}, \quad \text { for } M \geq M\left(\epsilon_{j}\right), 0<h \leq h\left(\epsilon_{j}, M\right),
$$

where

$$
I_{M}^{i}(v)=\int_{\Omega} f_{M}^{i}(x, v, D v) d x .
$$

(2) Let $M_{j} \geq M\left(\epsilon_{j}\right), 0<h_{j} \leq h\left(\epsilon_{j}, M_{j}\right)$. Let $u_{j}^{i} \in A_{h_{j}}(L)$ be minimizers of $I_{M_{j}}^{i}$ in $A_{h_{j}}(L)$. Then there exsit functions $\bar{u}^{i} \in A \cap W^{1, p}\left(\Omega ; R^{m}\right)$ and subsequences of $\left\{u_{j}^{i}\right\}_{j=1}^{\infty}$, again denoted by $\left\{u_{j}^{i}\right\}_{j=1}^{\infty}$, such that

$$
u_{j}^{i} \rightarrow \bar{u}^{i}, \quad \text { in } W^{1, p}\left(\Omega ; R^{m}\right),
$$

and

$$
I\left(\bar{u}^{i}\right)=\inf _{u \in A} I(u)=\underline{\lim }_{j \rightarrow \infty} I_{M_{j}}^{i}\left(u_{j}^{i}\right) .
$$

Proof. (1) follows from a similar argument as in lemma 3.1. (2) follows from a similar argument as in theorem 3.2. 
Remark. The arguments in the proof of lemma 4.1 show that the conclusions in lemma 4.1 and theorem 4.1 for $f_{M}^{1}$ hold without the hypothesis (iv).

Remark. Comparing the truncation method presented here with the element removal method [6], we see that both methods replaced the fast growth part of the integrand by certain slower growth functions so that the Lavrentiev phenomenon can be avoided. The difference is that in the element removal method the substitution is taken to be zero, while in the truncation method the truncation functions themselves, even though their growth is to a certain degree under control, still grow fast.

\section{$\S 5$. Numerical example}

I now apply the truncation method to the following 2-D problem, which is motivated by the 1-D problem of minimizing (1.1) in (1.2).

Take $\Omega=(-1,1) \times(0,1)$, and

$$
f\left(x, y, u, u_{x}^{\prime}, u_{y}^{\prime}\right)=h(x, y)\left(\left(u^{3}-x\right)^{2}\left(u_{x}^{\prime}\right)^{6}+\left(u_{y}^{\prime}\right)^{2}\right)
$$

where

$$
h(x, y)= \begin{cases}a y^{2}|x|^{3-b y^{2},} & \text { if } y \in(0,0.3) \\ 1, & \text { if } y \in[0.3,0.7] \\ a(1-y)^{2}|x|^{3-b(1-y)^{2}}, & \text { if } y \in(0.7,1),\end{cases}
$$

with $a=100 / 9, b=100 / 3$. Here $h(x, y)$ is so defined that for $y$ near either 0 or 1 the value of $h(x, y)$ goes to zero faster than $x^{2}$. This guarentees that the Lavrentiev phenonmenon still occurs in the problem.

Take

$$
\mathbf{A}=\left\{u \in W^{1,1}(\Omega): u( \pm 1, y)= \pm 1 ; u(x, y)=x, \text { on } y=0 \text { and } y=1\right\}
$$

Take $f_{M}=f_{M}^{2}$ defined by (4.4) with $\alpha_{M}=10^{-3} M, p=1.2$.

For $M=10$, devide $\Omega$ into rectangulars by introducing lines

$$
\begin{array}{r}
x_{i}=-1+\frac{i}{10}, 1 \leq i<20 \\
y_{j}=\frac{j}{10}, 1 \leq j<10 .
\end{array}
$$

Bilinear elements are used to construct the finite element function space $\mathbf{A}_{h}$. The numerical results of the truncation method and the standard finite element method are shown in Figure-1 and Figure- 2 respectively. 
For $M=20$, devide $\Omega$ into rectangulars by introducing lines

$$
\begin{gathered}
x_{i}=-1+\frac{i}{10}, \quad 1 \leq i<8 ; \\
x_{9}=-0.13, x_{10}=-0.08, x_{11}=-0.04, x_{12}=-0.02, \\
x_{13}=-0.01, x_{14}=0.0, x_{15}=0.01, x_{16}=0.02, \\
x_{17}=0.04, \quad x_{18}=0.08, x_{19}=0.13, \\
x_{i}=0.2+\frac{i-20}{10}, \quad 20 \leq i<28 ; \\
y_{j}=\frac{j}{10}, 1 \leq j<10 .
\end{gathered}
$$

Bilinear elements are used to construct the finite element function space $\mathbf{A}_{h}$. The numerical results of the truncation method and the standard finite element method are shown in Figure-3 and Figure-4 respectively.

Acknowledgment. I thank professor J.M. Ball and the referee for their valuable comments.

\section{REFERENCES}

[1] M. Lavrentiev, Sur quelques problèmes du calcul des variations, Ann. Math. Pure Appl. 4 (1926), 7-26.

[2] B. Manià, Soppa un esempio di Lavrentieff, Bull. Unione Mat. Ital. 13 (1934), 147-153.

[3] J. M. Ball \& V. J. Mizel, One-dimensional variational problems whose minimizers do not satisfy the Euler-Lagrange equation, Arch. Rat. Mech. Anal. 90 (1985), 325-388.

[4] J. M. Ball \& G. Knowles, A numerical method for detecting singular minimizers, Numer. Math. 51 (1987), 181-197.

[5] A. M. Davie, Sigular minimizers in calculus of variations in one dimension, Arch. Rat. Mech. Anal. 101 (1988), 161177.

[6] Z. P. Li, Element removal method for singular minimizers in variational problems involving Lavrentiev phenonmenon, Proc. R. Soc. Lond. A 439 (1992), 131-137.

[7] Z. P. Li, A theorem on lower semicontinuity of integral functionals (to appear).

[8] P. G. Ciarlet, The Finite Element Method for Elliptic Partial Differential Equations, North-Holland, Amsterdam, 1978. 